\title{
Delayed Diagnosis of Oral Malignancy and Effect to Prognosis; Case Series
}

\author{
Yuli Fatzia Ossa ${ }^{1, *}$, Citra Feriana Putri ${ }^{2}$ \\ Department of Oral Medicine, Faculty of Dentistry, Universitas Syiah Kuala, Banda Aceh, Indonesia \\ Department of Oral Biology, Faculty of Dentistry, Universitas Syiah Kuala, Banda Aceh, Indonesia \\ Corresponding author email: yulifatziaossa@unsyiah.ac.id
}

\begin{abstract}
Introduction: Oral cancer is one of most common cancer in the world. Oral cancer can affect any part of oral cavity and oropharyngeal. Etiology of oral cancer is multifactorial. Delay in diagnosis and management of oral cancer can worsen of prognosis and increase case mortality. Here we report 3 cases of delayed diagnosis oral cancer. Case Report: First case, patient with history of persistent oral ulcer for 2 years, patient has gone to many doctors but the ulcer does not healed. The second case, patient with persistent oral ulcer for the last 1 year, and the third case, patient with history of persistent oral ulcer for 5 month without improvement. All these patients referred to oral medicine specialist, and when the oral examination was performed, they are diagnosed with suspected oral squamous cell carcinoma and then we referred to oncologist for further examination. Discussion: Oral squamous cell carcinoma is most common oral cancer with survival rate at earlier stages relatively good than advanced stage. Diagnostic delay in oral cancer is one of main reason the patients are in advanced stage when their diagnosed are finally made. In our cases, all of the patients had persistent oral ulcer with the diagnosis is oral cancer and we suggest all of them are in the advanced stage of oral cancer. Conclusion: Oral cancer can be prevented by early detection of the lesions. It is important to educate patient and public to be more knowledgeable about oral cancer, and also to general practitioner.
\end{abstract}

Keywords: Delayed Diagnostic, Oral Cancer, Squamous Cell Carcinoma, Screening, Early Detection 


\section{INTRODUCTION}

Oral cancer can occur in any part of oral cavity. Squamous cell carcinoma is the most common type of oral cancer, and is ranked $6^{\text {th }}$ of cancer around the world based on the incidence oral cancer data in 2016. ${ }^{1}$ Data from the World Health Organization (WHO), there is an increase of 300 thousand new cases of oral cancer in worldwide and still counting. $50 \%$ cases incidences occurring in ASIA, $11 \%$ cases are from Southeast-ASIA including Indonesia. In Indonesia, based on Riset Kesehatan Dasar (Riskesdas) 2018, an increased the number of oral cancer about $5.6 \%$ compared to data in 2013 which was $1.4 \% .^{2}$

Early diagnosis of oral cancer greatly affects the patient prognosis and survival. The survival rate in the early stages of the disease is around $84 \%$, while in the advanced stages it drops drastically to $9 \%$. The prognosis in the early stages of disease relatively good, unfortunately, 40-60\% of patients are at an advanced stage when clinical examination is performed. ${ }^{3,4}$ In most countries, predilection of oral cancer more common in men than women/ it is associated with the risk of habits, such as smoking and alcohol consumption which are more common in man, as well as exposure to sunlight for lip cancer. Poor diagnosis of oral cancer is generally seen on clinical examination at the late stage of disease. Patients usually delay seeking professional advice. The average patients delays for 3 month after realizing the symptoms that occur in oral cavity which related with oral cancer. ${ }^{5}$ Here we reported 3 cases delayed diagnosis of oral cancer, and we would discuss about delayed diagnosis and molecular pathologist of oral cancer.

\section{CASE REPORT}

- First Case

A 38 year old woman with complaints of oral ulcer on the right side of the tongue, it is appeared 2 years ago due to sharp teeth. Lesion asymptomatic, she already visited general practitioner about one year ago, but the lesion still persist. She also went to Ear Nose and Throat Specialist, and got the antifungal as a treatment of lesion, but also no change. Then, she was referred to oral medicine specialist. When we do the anamnesis there is significantly weight loss, she denied any history of malignancy in the family. Extra-oral examination, sub-mandibular and sub-mental lymph nodes fixed and painless. Intra-oral examination, an exophytic tumor with ulceration at the lateral edge of the right tongue. Ulcer indurated, with irregular margin. There is non-homogeneous white plaque contiguous ulcer that cannot be rubbed off. At the time of examination, we diagnosed lesion as tongue cancer, then were referred to oncologist for biopsy and follow up examination (Figure.1).

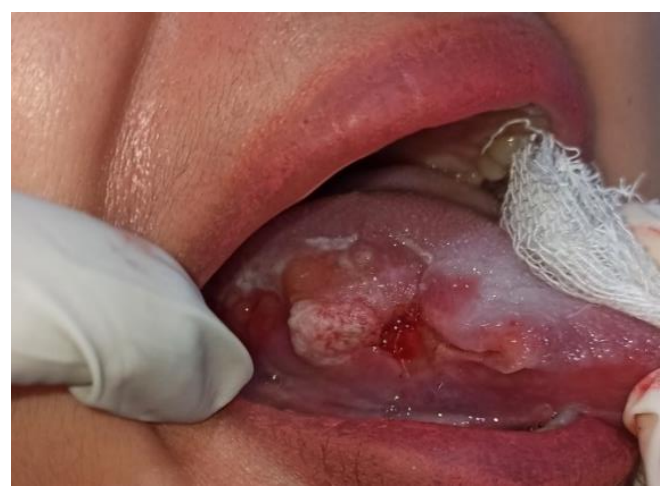

Figure.1. Ulcer on the lateral edge of the tongue

- Second Case

A 51 year old woman went to oral medicine specialist with complaint of persistent oral 
ulcer for past 1 year. Ulcer on the left side of tongue, started out small ulcer than it grows. She has tried to treat this lesion by going to visit general practitioners, and it treated with antifungal but there was no improvement. She has history of recurrent oral ulcer and history of malignancy was denied. At the time of extra-oral examination, the left sub-mental lymph node palpable, fixed, with pain. Enlargement of cervical lymph node on left side with diameter about $7 \mathrm{~cm}$, palpation was fixed and painless. Intraoral examination found widespread ulceration accompanied by an exophytic mass on the lateral edge of left tongue. Non-homogenous plaque on posterior and white plaque on ventral left of the tongue. Ulcer indurated, tongue movement is limited, the lesion extended through the midline of the dorsal tongue. We confirmed the diagnosis as oral squamous cell carcinoma, then patient referred to oncologist for biopsy and further examination and treatment (Figure 2 and 3).

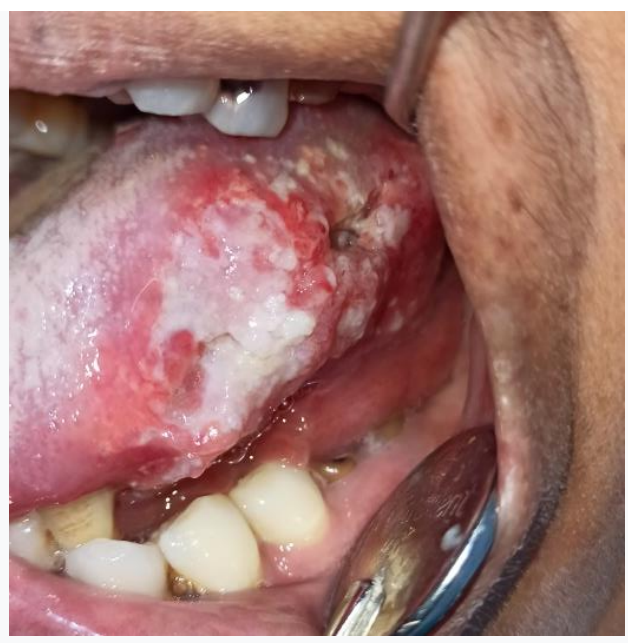

Figure 2. Large ulcer on lateral of tongue

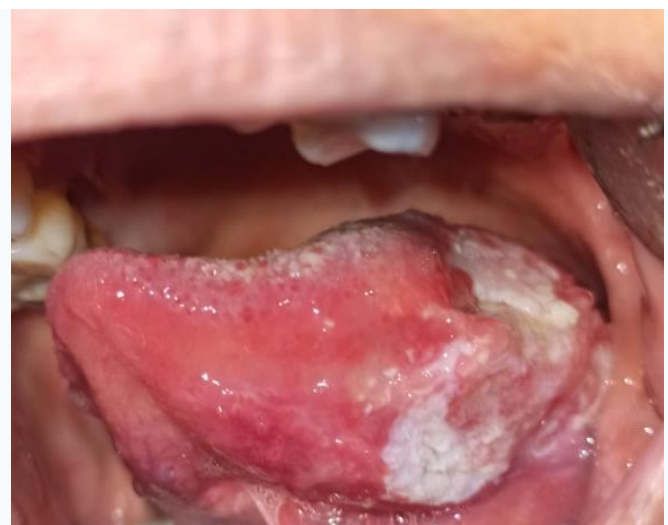

Figure 3. Oral Ulcer on the left side lateral tongue with non homogenous plaque on ventral

\section{- Third Case}

A 48 age years male patient with chief complain of persistent oral ulcer for 5 month, ulcer have been treated, but there was no improvement, he applied antifungal, gentian violet to the ulcer. History of smoking since 2002, history of malignancy in family was denied, history of recurrent oral ulcer. Extraoral examination sub-mental lymph node was palpable, firm and painless. Intra-oral examination revealed oral ulcer on the left lateral edge of the tongue, deep ulcer with raised edges, irregular, indurated. There were no traumatic causes around the ulcer (Figure $4)$.

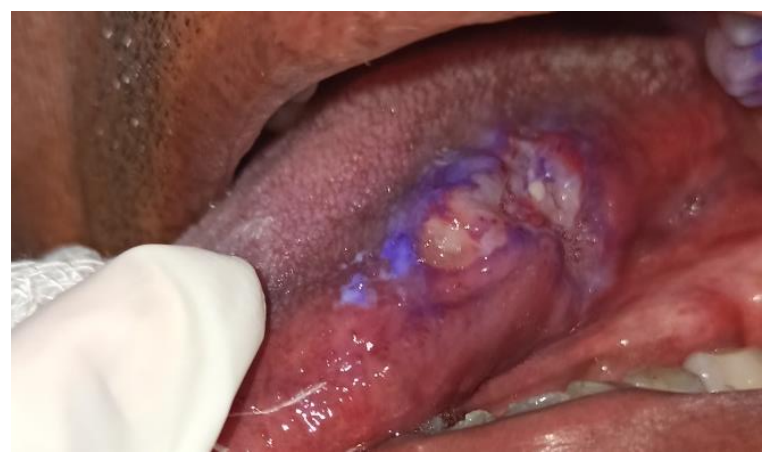

Figure 4. Oral ulcer on the left lateral tongue 


\section{DISCUSSION}

Etiology of oral cancer are multifactorial such as, smoking cigarette, tobacco, alcohol, betel nut chewing, genetic, UV light, and human papilloma virus infection. Men more common affected because of smoking cigarette, alcohol abuse, and tobacco used. Most case of oral cancer develop in people aged 45-60 years old. ${ }^{6}$ In our cases, one of patient has smoking habit more than 10 years, and the others denied having any habit associated with oral cancer predisposing factors. They also denied any history of cancer in other family members.

Clinically, oral cancer may manifest as an irregular ulceration accompanied by raised margins of the lesion and the presence of induration, in addition, the lesions may also have the appearance of areas of nonhomogeneous plaque. The most common sites are the posterior lateral of the tongue, floor of the mouth, lips, and oropharynx. ${ }^{7}$ In our cases, the lesions located on the lateral edge of the tongue, ulceration with exophytic masses, and areas of non-homogenous plaque.

Pain in oral cancer conditions can be the first complaint and can occur in $66 \%$ of cases of oral cancer, and pain is also the initial reason for patient to seek treatment. The most important thing is patient are often unaware of the appearance of oral cancer, especially at the early stage. This causes patient to be late in seeking for treatment. Commonly pain doesn't appear in the early stages until several months later after the clinical finding. ${ }^{4,8}$ In our cases, all of the patients experienced oral discomfort several month after the lesion appeared, and the lesion was no improvement with any treatment.
As with the nature of cancer in general, prognosis of oral cancer depends on the extent of the cancer and the initial diagnosis and initiation of treatment. Prognosis factors for oral cancer include tumor size and stage of cancer, lymph node involvement, distant metastases, and the most importantly the stage of cancer at the time the lesion was first discovered. Another factor is location of lesion. The location of the posterior area shows worsen prognosis than other location in the oral cavity. It is because generally lesion on the posterior area is difficult to detect at initial screening. ${ }^{9,10}$ The prognosis of oral cancer if it can be diagnosed at an early stage (I and II) is generally moderate to good, with an estimated will live for 5 at last 5 years after diagnosis. (70\%-90\% of people with earlystage oral cancer). Meanwhile, if oral cancer diagnosed at an advanced stage (III and IV), prognosis is poor (only one in five people will live for at least 5 years after diagnosis). ${ }^{8,11}$ The most effective to prevent oral cancer is to manage lifestyle, avoid risk factor, and eat healthy diet. For those affected by oral cancer, early detection by screening may improve chances of survival rate. ${ }^{1}$

In our case report, 2 of the patients had lymph node involvement in the head and neck this was obtained during lymph node examination. Examination of the lymph nodes, cervical and sub-mandibular lymph nodes are palpable, firm, and fixed. This is a marker of cancer development. The growth pattern of oral cancer is relatively long and does not metastasize in the first few months of clinical symptoms. However, if oral cancer delayed in diagnosis, it will keeps growing and can interfere oral function such as speaking, chewing, and also impact to quality of life. 
Early detection, as well as immediate management can minimize the side effect of cancer. $^{12}$

Survival rates for early stage of oral cancer approximately $84 \%$ while for advanced- stage it drop significantly to $39 \%$. Treatment of oral cancer at an early stage is believed to be more effective in reducing mortality, morbidity, and disability. The prognosis for early stage is relatively good, however more than $50 \%$ of patient with advanced stage of oral cancer on presentation. ${ }^{13,14}$

\section{Molecular Pathogenesis of Oral Cancer}

Genetic and environmental factors are known to have an important role in modulating the process of carcinogenesis. Genetic factors play a role in the disruption of normal regulatory mechanisms that control basic cellular functions of the body consisting of cell division, cell differentiation and cell death. Hanahan and Weinberg have described Hallmark 1 and Hallmark 2 of cancer, namely: Hallmarks 1 consists of disturbances in autonomic cell growth signals (oncogenes), interference with growth inhibitory signals (tumor suppressor genes), avoidance of apoptosis, cell death, angiogenesis, invasion and metastases. Meanwhile, Hallmarks 2 consists of reprogramming energy metabolism and avoiding immune responses, genome instability and mutations, and tumorpromoting inflammation. ${ }^{15}$

Molecular pathogenesis related to oral carcinoma is known to occur due to chromosomal changes, p53 and GLUT-1 epigenetic disorders, inhibition of immune system function, the role of oxidative stress, and the involvement of the Epstein-Barr Virus (EBV).

\section{Chromosomal Changes}

In the process of oral squamous carcinogenesis, there are multiple genetic disorders of the normal functioning of oncogenes and tumor suppressor genes. Although, the involvement of the two is still not known with certainty. Histological changes in oral carcinogenesis from hyperplasia to dysplasia, followed by severe dysplasia and the occurrence of invasion and metastasis are due to genetic changes as shown in the following diagram. (Picture 4). The diagram shows the occurrence of genetic changes from dysplasia to Oral squamous cell carcinoma (OSCC), due to changes in the p or q arms on chromosomes $3,4,8,9,11,13$, and $17 .{ }^{16}$

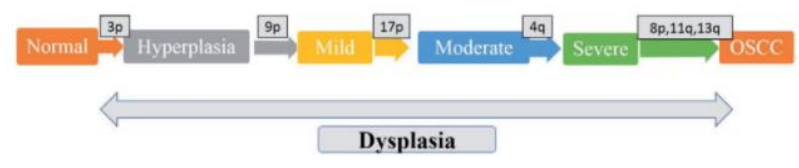

Picture 4. Molecular model of oral carcinogenesis ${ }^{16}$

\section{Epigenetic disorders of p53 and GLUT-1}

The protein encoded by the p53 gene is a transcription factor and has a function in controlling the initiation of the cell cycle. Signal transduction mediated by p53 is very important in regulating normal cell activity. After a mutation, the p53 gene loses its ability in cell growth, apoptosis and DNA repair, and p53 changes from a tumor suppressor gene to an oncogene. Several studies have shown that there is overexpression of $\mathrm{p} 53$ protein in 
patients with premalignant lesions and oral malignant lesions. ${ }^{17}$

Glucose transporters (GLUTs) are proteins that mediate glucose transport across cell membranes. Glucose metabolism depends on the uptake of glucose by cells. However, glucose cannot enter the cell freely through the lipid bilayer structure of the cell membrane. Absorption of glucose into cells requires a glucose transporter called GLUT. In the case of malignant cells, it is known that there is an overexpression of GLUT-1, which allows the cells to experience increased proliferative activity, energy requirements, more aggressiveness, and poor response to radiation. GLUT-1 expression was strongly correlated with the histological grade and pathology of Tumor Node Metastasis (pTNM) staging of OSCC. ${ }^{18}$

\section{Inhibition of immune system function}

The presence of immune dysfunction plays a role in the success of cancer cells in avoiding the function of the immune system, triggering the formation of new cancer cells, and the development of cancer cells becomes more severe. IL-4 is an anti-inflammatory cytokine known to have anti-tumor activity in breast and colon cancer. In a 2010 study investigating systemic immunity and the expression of IL-4 and IL-2 on T cells from peripheral blood of patients with OSCC. The results showed that there was a decrease in CD4+ IL-2 and CD8+ IL-4+, as well as CD3+ IL-4+ T cells. ${ }^{18}$

\section{The Role of Oxidative Stress}

Free radicals such as reactive oxygen species (ROS), reactive nitrogen species (RNS) and reactive oxygen metabolites such as $\mathrm{H} 2 \mathrm{O} 2, \mathrm{O} 2-, \mathrm{OH}-, \mathrm{NO}$, and malondialdehyde can induce DNA damage and DNA chain breakdown, DNA cross linkage, and base modification. DNA. It can initiate and promote carcinogenesis. These free radicals can also interfere with the antioxidant system. GSH protein is an indicator of the protection of organisms from the toxicity and detoxification of hydrogen peroxide. However, in cancer, the oxidant was found to be too high while the antioxidant activity of GSH was very low so that it became the cause of the progression of cancer. ${ }^{18}$

\section{Presence of Eipstein-Barr Virus (EBV) involvement}

Eipstein-Barr Virus causes lifelong persistent infection in more than $90 \%$ of the world's population. The virus has an incubation period in healthy individuals who carry the virus. Under ambient pressure, the virus can be reactivated periodically during the lifetime of the individual. The reactivation of this virus can be triggered by smoking tobacco. The presence of EBV is known to be associated with malignancies such as Burkit's lymphoma, Hodgkin's disease, stomach cancer, and nasopharyngeal carcinoma (NPC), and OSCC. ${ }^{18}$

\section{Delayed Diagnosis of Oral Cancer}

In some literatures reported the delay in diagnosis of oral cancer can be influenced by the patient's delay and professional delay. Patient's delay is defined as the length of time from the time she or he aware of symptoms of lesions until the time she or he visits physician or general practitioner. Often this time is approximate because patients tend to estimate the time between time in month, sometimes 
they can identify the days and weeks when they first notice about lesion. Professional delay, generally defined as the time between the patient's initiation of professional care and time to start of treatment. ${ }^{14,19,20}$

The gap between time the patient was examined by a specialist and the time when patient is first admitted. In our cases, the patient with history of oral lesions since 2 years, she has tries to see a general practitioner, and ENT specialist, but there was no improvement. In this case, it was a delay in establishing the diagnosis. When the patient came to oral medicine specialist, the diagnosis of oral cancer was made. The second patient also took a very long time until the diagnosis of oral cancer was made, which was 1 year from the onset of symptoms. While the third patient, diagnosis of oral cancer was established 5 month after the initial symptoms appeared. The delay in seeking treatment to a specialist is that they feel that lesions in theit oral cavity are common things. Besides, when we do anamnesis, they said they have history of recurrent oral ulcer, so this made the patient delay seeking treatment.

Patient's delay in seeking treatment related to oral cancer can be influenced by the lack of knowledge of oral lesions that have potentially transformed to oral cancer. Routinely oral screening can help the patient more aware of changes of the oral cavity. Self oral cancer screening may be able of identify an early change in oral cavity. Oral pre cancer and cancers are looks like; white spots with sharply defined borders from normal tissue; red spot with sharply defined borders from the normal tissue; ulcers that do not heal in 2 weeks; and lumps or bumps growing out of the tissue. ${ }^{21}$

\section{CONCLUSION}

Oral cancer can be prevented by early detection of the lesions. The prognosis of oral cancer in early stage approximately good, while for advanced- stage it worsen the prognosis. Treatment of oral cancer at early stage is believed to be more effective in reducing mortality, morbidity, and disability. The prognosis for early stage is relatively good, however more than $50 \%$ of patient with advanced stage of oral cancer on presentation. It is important to educate patient, public, and also general practitioner to be more knowledgeable about oral cancer, so that patient delay and professional delay should be avoided.

\section{ACKNOWLEDGMENT}

We would like to thank all patients in this case report.

\section{CONFLICT OF INTEREST}

There are no conflicts of interest

\section{REFERENCES}

1. Warnakulasuriya S, Fennell N, Diz P, et al. An appraisal of oral cancer and precancer screening programmes in Europe: A systematic review. J Oral Pathol Med. 2015;44(8):559-570. doi:10.1111/jop.12267

2. Kemenkes RI. Hasil Riset Kesehatan Dasar Tahun 2018. Kementrian Kesehat RI. 2019;53(9):1689-1699.

3. Gigliotti J, Madathil S, Delays NM, Oral IJ. Delays in oral cavity cancer. Int J Oral Maxillofac Surg. 2019;48(9):1131-1137. doi:10.1016/j.ijom.2019.02.015

4. Brekel MWM Van Den. The association of treatment delay and prognosis in head and neck squamous cell carcinoma 
( HNSCC ) patients in a Dutch comprehensive cancer center q. Oral Oncol. 2014;50(4):282-290. doi:10.1016/j.oraloncology.2013.12.018

5. Warnakulasuriya S. Living with oral cancer: Epidemiology with particular reference to prevalence and life-style changes that influence survival. Oral Oncol. 2010;46(6):407-410. doi:10.1016/j.oraloncology.2010.02.015

6. Warnakulasuriya S. Oral potentially malignant disorders: A comprehensive review on clinical aspects and management. Oral Oncol. 2020;102(May 2019):104550. doi:10.1016/j.oraloncology.2019.10455 0

7. Rhodus NL, Kerr AR, Patel K. Oral Cancer. Leukoplakia, Premalignancy, and Squamous Cell Carcinoma. Dent Clin North Am. 2014;58(2):315-340. doi:10.1016/j.cden.2013.12.004

8. Ganly I, Patel S, Shah J. Early stage squamous cell cancer of the oral tongueclinicopathologic features affecting outcome. Cancer. 2012;118(1):101-111. doi:10.1002/cncr.26229

9. Akbulut N, Oztas B, Kursun S, Evirgen S. Delayed diagnosis of oral squamous cell carcinoma: A case series. $\mathrm{J}$ Med Case Rep. 2011;5:2-5. doi:10.1186/1752-1947-5-291

10. Saka-Herrán C, Jané-Salas E, MariRoig A, Estrugo-Devesa A, LópezLópez J. Time-to-treatment in oral cancer: Causes and implications for survival. Cancers (Basel). 2021;13(6):113. doi:10.3390/cancers13061321

11. Rogers SN, Brown JS, Woolgar JA, et al. Survival following primary surgery for oral cancer. Oral Oncol. 2009;45(3):201-211.

doi:10.1016/j.oraloncology.2008.05.008

12. Douglas E Peterson NLR. Oral Cancer. In: Orofacial Disorders. Springer International Publishing; 2017:15-20. doi:10.1007/978-3-319-51508-3_2

13. Scott SE, Grunfeld EA, McGurk M. Patient's delay in oral cancer: A systematic review. Community Dent Oral Epidemiol. 2006;34(5):337-343. doi:10.1111/j.1600-0528.2006.00290.x

14. Jafari A, Najafi S, Moradi F, Kharazifard M, Khami M. Delay in the diagnosis and treatment of oral cancer. $J$ Dent (Shiraz, Iran). 2013;14(3):146150.

http://www.ncbi.nlm.nih.gov/pubmed/2 4724136\%0Ahttp://www.pubmedcentra 1.nih.gov/articlerender.fcgi?artid=PMC3 927673

15. Hanahan D, Weinberg RA. Hallmarks of cancer: The next generation. Cell. 2011;144(5):646-674. doi:10.1016/j.cell.2011.02.013

16. Daaboul H, ed. Squamous Cell Carcinoma-Hallmark and Treatment Modalities.; 2020.

17. Jiang $\mathrm{X}, \mathrm{Wu} \mathrm{J}$, Wang $\mathrm{J}$, Huang $\mathrm{R}$. Tobacco and oral squamous cell carcinoma: A review of carcinogenic pathways. Tob Induc Dis. 2019;17(April):1-9. doi:10.18332/tid/105844

18. Lai PEIC, Chiu TEDH, Huang YENTA. Overexpression of BDNF and TrkB in human. Anticancer Res. 2010;31(12):1265-1270. doi:10.3892/or

19. Gao W, Guo C Bin. Factors Related to Delay in Diagnosis of Oral Squamous Cell Carcinoma. J Oral Maxillofac Surg. 2009;67(5):1015-1020. doi:10.1016/j.joms.2008.12.022

20. Tsai WC, Kung PT, Wang YH, Huang $\mathrm{KH}$, Liu SA. Influence of time interval from diagnosis to treatment on survival for oral cavity cancer: A nationwide cohort study. PLoS One. 2017;12(4):114. doi:10.1371/journal.pone.0175148

21. Ford PJ, Farah CS. Early detection and diagnosis of oral cancer: Strategies for improvement. $J$ Cancer Policy. 2013;1(1-2):e2-e7. doi:10.1016/j.jcpo.2013.04.002 